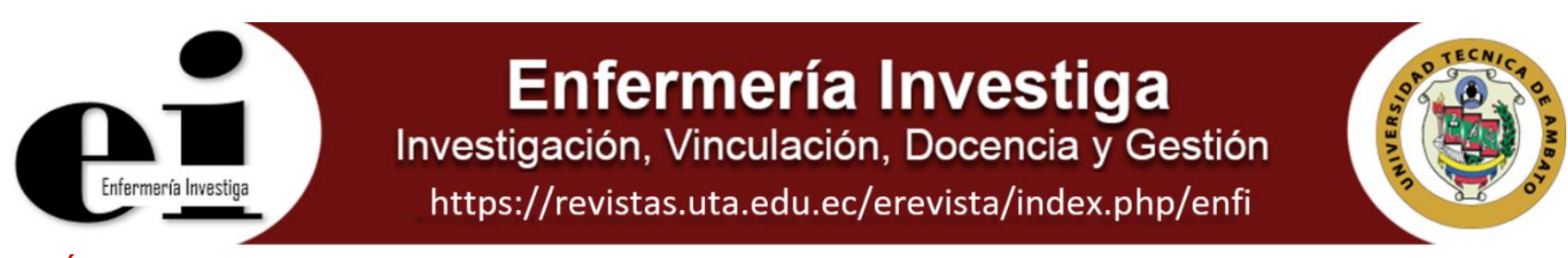

\title{
DIMENSIÓN ESPIRITUAL EN EL CUIDADO ENFERMERO
}

\section{SPIRITUAL DIMENSION IN NURSING CARE}

Blanca Nivia Morales Contreras ${ }^{1}$ https://orcid.org/0000-0003-3950-1729, Jenyfer Jessika Palencia Sierra1. https://orcid.org/00000001-7675-6304

\author{
${ }^{1}$ Programa Enfermería. Universidad Popular del Cesar. Colombia.
}

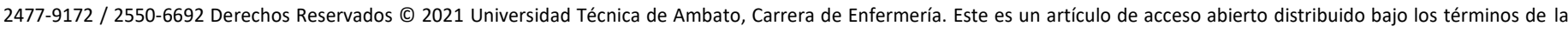
Licencia Creative Commons, que permite uso ilimitado, distribución y reproducción en cualquier medio, siempre que la obra original es debidamente citada.
\end{abstract}

\section{RESUMEN}

Para los profesionales de enfermería, el cuidado de la dimensión espiritual ha sido un continuo reto para asumir en el ciclo vital completo y en todas las áreas del desempeño en las cuales enfermería es responsable; la espiritualidad como esencia del cuidado de todo ser humano, debe ser integral con las otras dimensiones, en una reciprocidad entre la persona de cuidado y el profesional. Este artículo tiene como objetivo revisar la literatura del cuidado brindado por el profesional de enfermería en la dimensión espiritual; categorizar y analizar las intervenciones de enfermería de acuerdo a la revisión bibliográfica y proponer acciones desde el arte del cuidado de enfermería. La reflexión del equilibrio dinámico de la vida humana y la interacción con el entorno y los constantes cambios que como personas enfrentamos, origina la necesidad de tener mayor claridad en los aspectos prácticos para el cuidado de la dimensión espiritual, con base en la premisa que la persona es un ser espiritual por la esencia de su naturaleza, por estas razones la dimensión espiritual se debe integrar con la dimensión corporal, social, cognitiva, comunicativa, estética, emocional y ética.

Palabras claves: Arte y cuidado de enfermería, dimensión espiritual, ética del cuidado.

\section{ABSTRACT}

For nursing professionals, caring for the spiritual dimension has been a continuous challenge to assume throughout the entire life cycle and in all areas of performance in which nursing is responsible; spirituality as the essence of the care of every human being, must be integral with the other dimensions, in a reciprocity between the caregiver and the professional. This article aims to review the literature on the care provided by the nursing professional in the spiritual dimension; categorize and analyze nursing interventions according to the bibliographic review and propose actions from the art of nursing care. The reflection of the dynamic balance of human life and the interaction with the environment and the constant changes that we as people face, originates the need to have greater clarity in the practical aspects for the care of the spiritual dimension, based on the premise that the person is a spiritual being by the essence of his nature, for these reasons the spiritual dimension must be integrated with the corporal, social, cognitive, communicative, aesthetic, emotional and ethical dimension

Keywords: Nursing art and care, spiritual dimension, ethics of care.

Autor de correspondencia: PhD Mg. Blanca Nivia Morales Contreras. Correo electrónico: blanca.n.morales@gmail.com

\section{INTRODUCCIÓN}

A través del tiempo los profesionales de enfermería han reflexionado, sobre el cuidado en la dimensión espiritual, con diferentes enfoques frente a las definiciones que hay de la espiritualidad; asumiendo la responsabilidad en la integralidad del cuidado (1) para las personas, familias, comunidades y una sociedad pensante, analítica, reflexiva que demanda cada vez más cuidados en las diferentes dimensiones del ser humano, de manera especial en lo espiritual.

La espiritualidad está inmersa en la persona, favorece el encuentro, la unión, la trascendencia con su ser creativo, con el medio ambiente, con su expresión energética, con la esencia como ser universal. La espiritualidad emerge desde el mismo momento que hay vida, en el crecimiento del SER va cambiando, se va perfeccionando, se va interiorizando como una forma de diálogo interno, donde la persona busca estar en concordancia con los valores, las creencias y las culturas. La espiritualidad permite entender el pensamiento religioso de muchas culturas, donde existe un ser supremo llamado Dios, que les guía, les da la ruta de vida, entendido dentro del marco de múltiples religiones que ayudan al hombre a trascender. Lo cual, es indiscutible porque "vivimos en una sociedad plural, pero esto no significa afirmar que vivimos en una sociedad pluralista, esto es, 
que cada uno tenga su verdad" (3). "El integrarla, conlleva un crecimiento personal, la sanación y búsqueda de la armonía de la mente, cuerpo y espíritu en las personas" (2). La espiritualidad inmersa en "la ciencia es una iluminación de Dios y del universo en la mente del hombre, para avanzar y vivir en paz. Convirtiéndose, en arte cuando alcanza a mirar el producto de la vivencia por la recreación personal. Es decir, la espiritualidad es arte porque es la ciencia más un don" (4).

La definición de espiritualidad es reflexionada por diferentes investigadores, ha sido dimensionada en tres aspectos 0 perspectivas: una de origen intrínseco dada desde la persona misma, sus pensamientos, creencias, desarrollo de vida. Otra perspectiva religiosa, que permite ampliar las normas y poder relacionarse con el sí mismo, las personas y el infinito. Por último, se habla de una perspectiva existencial donde el ser humano está en una constante indagación de la importancia de la coexistencia, de la vida, se reflexiona como individuo, como un reflejo del otro. Desde el cuidado integral es imprescindible vislumbrar al ser humano con una anatomía física, una mente y espíritu en constante trascendencia, fomentando una salud en estrecha integridad (5).

Desde los cuidados paliativos (6), se tiene mayor apropiación teórica en el cuidado de la dimensión espiritual por parte del profesional de enfermería. Sin embargo, es relevante responsabilizar a enfermería en el fomento del cuidado espiritual en todas las etapas del ciclo vital, hacer intervención teniendo en cuenta la historia natural de la enfermedad y los niveles de prevención (7). Se deben establecer más estrategias para llegar con el cuidado espiritual a todas las poblaciones, sanas o enfermas a fin de lograr una salud óptima en lo individual, familiar y colectivo. La Organización Mundial de la Salud (OMS), en 1990 en su definición de cuidados paliativos acoge la dimensión espiritual, relevante para ampliar los conceptos de salud y enfermedad que se tienen en la actualidad, siendo "el cuidado activo y total de los pacientes portadores de enfermedades que no responden a tratamiento curativo [enfatizando que] el control del dolor y de otros síntomas, así como la atención de aspectos psicológicos, sociales y espirituales, es primordial" (8).

Es por ello, que la reflexión de este artículo nace de una revisión de antecedentes bibliográficos, evidenciando interés y reflexión de los profesionales de enfermería frente a la temática

\section{DESARROLLO}

La espiritualidad es definida por la European Association of Palliative Care (EAPC) como "la dimensión dinámica de la vida humana que se relaciona con el modo en que las personas (individuo y comunidad) experimentan, expresan y/o buscan significado (9), propósito (10) y trascendencia, y el modo de conectarse con el momento, el 'yo', los otros, la naturaleza, lo significativo y/o lo sacro" $(6,11)$. La espiritualidad está dispuesta de manera inherente al ser humano, a la familia, comunidad y sociedad, tiene en cuenta principios morales relevantes para un desarrollo integral y eficaz (12). Se afirma que la espiritualidad es universal, cuando el individuo la conoce, su naturaleza mental es superior, asume de mejor manera la salud en lo corporal y comunicativo; se evidencia una alta capacidad de afrontar las enfermedades (13). "En un estudio Terceño (2017) distinguió dos maneras de abordar los cuidados espirituales por parte de enfermería, una estaba relacionada con la religión y la otra que tenía que ver con el conocimiento interno de la persona.
Concluyendo que, aunque sean maneras diferentes de entender los cuidados ambas eran perfectamente compatibles" (11). Además, Sandoval (2014) demuestra que el concepto religiosidad obtuvo mayor relevancia, frente a la espiritualidad para el equipo multidisciplinario de cuidados paliativos de una institución de salud, se analiza que algunas de las palabras son referentes sinónimos de religión y espiritualidad, ejemplo: Dios, religión, amor (14). Por otro lado, es importante señalar que los profesionales de enfermería deben empatizar con su autocuidado y el cuidado de otros desde lo espiritual, con una personalidad de legitima ayuda, entrega, disposición, tolerancia, misericordia, humanidad, presencia consciente, responsable y científica (15).

Gutiérrez (2020) ha reflexionado la espiritualidad desde lo bidimensional, mostrando una dimensión vertical, donde hay un ser superior, un Dios que gobierna la vida de la persona; una dimensión horizontal donde el individuo se relaciona con su mundo interno, con la familia y el entorno (16). Para Suárez (2015), las necesidades espirituales "son el vigor natural y la virtud que alienta, fortifica, da fuerza y valor para actuar en el medio social al cual pertenece la persona" (17). Es por ello, que para el profesional de enfermería es un reto el cuidado espiritual porque puede influir en el aspecto emocional, de lo ético, de lo comunicativo, en la interacción de lo integral. Razón por la cual, es importante tener en cuenta algunas características de la espiritualidad, para potenciar la salud individual, resaltando lo planteado por Alarcón (2009) (18), para "revisar la relación con uno mismo, ¿me tengo confianza?, ¿me pienso?, ¿me reflexiono como persona?, ¿qué conocimiento tengo de mí mismo?, ¿confió en la vida?, ¿confió en mi familia?, ¿cómo es mi relación con la naturaleza, con el medio ambiente?, ¿me siento en paz, en armonía?, ¿cómo me relaciono con mi pareja, con mi entorno?, ¿tengo conflictos con los demás?, ¿me relaciono con algún ídolo?, ¿soy religioso o no?, ¿hago oración, meditación?, ¿me relaciono con mi comunidad religiosa?, ¿tengo contacto directo con la naturaleza?.

Por lo anterior, el profesional de enfermería, como eje central en el cuidado, propende por ser líder en la valoración, diagnóstico, planeación, ejecución y seguimiento a la atención espiritual, en todo el ciclo vital, no solamente en los casos en que la persona requiere cuidados paliativos; o al final de la vida. Trabaja en forma armónica, respetuosa y humanizada de la mano con el equipo interdisciplinario a fin de lograr metas que conlleven a la mejoría de las necesidades espirituales de la persona, familia y comunidad; con una visión espiritual acorde a las necesidades sentidas por todo ser humano, sensible al cuidado de la naturaleza, del contexto físico y emocional (19).

El profesional de enfermería, sabe que si una persona no cubre sus necesidades espirituales sufre, manifestando de diferentes maneras su sentir, eso depende de la forma como se relacione consigo mismo, con los otros, con el entorno, la naturaleza, el arte, para así recuperar su paz interior y su salud desde donde se conciba, ya sea en enojo, alienación o desesperanza (20). Finalmente, enfermería puede apoyarse de la logoterapia (21) que centra su hacer en encontrar sentido a la vida, donde las personas pueden dialogar y expresar lo más profundo de su ser, asumiendo que cada persona es única e irrepetible (22). El profesional de enfermería trasciende lo corporal, se preocupa entre otras dimensiones por la dimensión espiritual, al tratar de encontrar esa singularidad del SER desde lo individual, familiar, comunitario, confrontando de manera interior su propia dimensión espiritual. A continuación, se hace referencia a los 
principales modelos y teorías de enfermería (23) que han resaltado la dimensión espiritual en la práctica del cuidado:

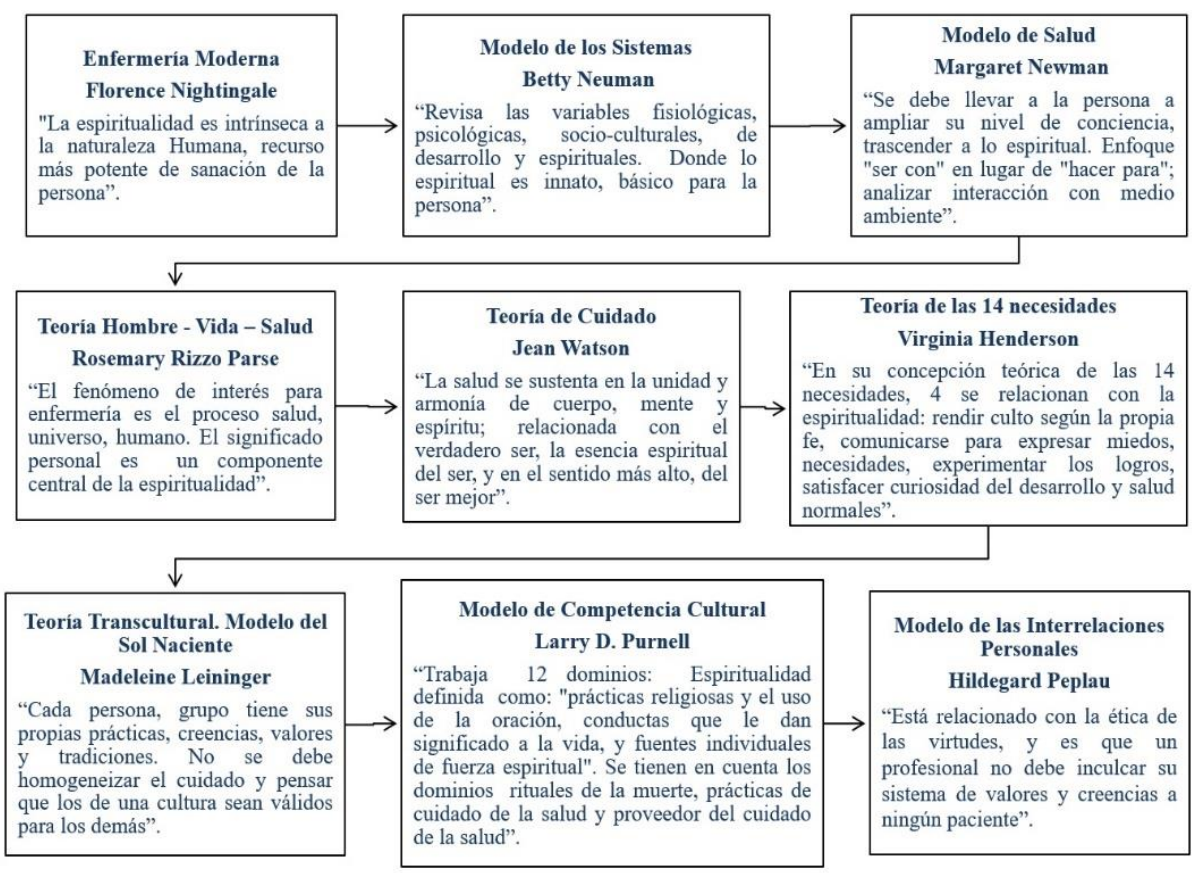

\section{llustración 2. Adaptado de: Marriner, Tomey. (2011). Modelos y teorías en enfermería.}

Sumado a lo anterior, en el arte del cuidado, enfermería desde su intervención interrelaciona las dimensiones humanas (24) para que con justa prudencia pueda discernir los puntos de encuentro que beneficiaran a la persona, familia y/o comunidad que cuida, al dominar la técnica del cuidado trasforma, porque es capaz de ver la belleza, la estética, la esencia, el origen, los puntos de unicidad del encuentro que hace que lo diferente se interrelacione comunicando un lenguaje que va más allá de las palabras.

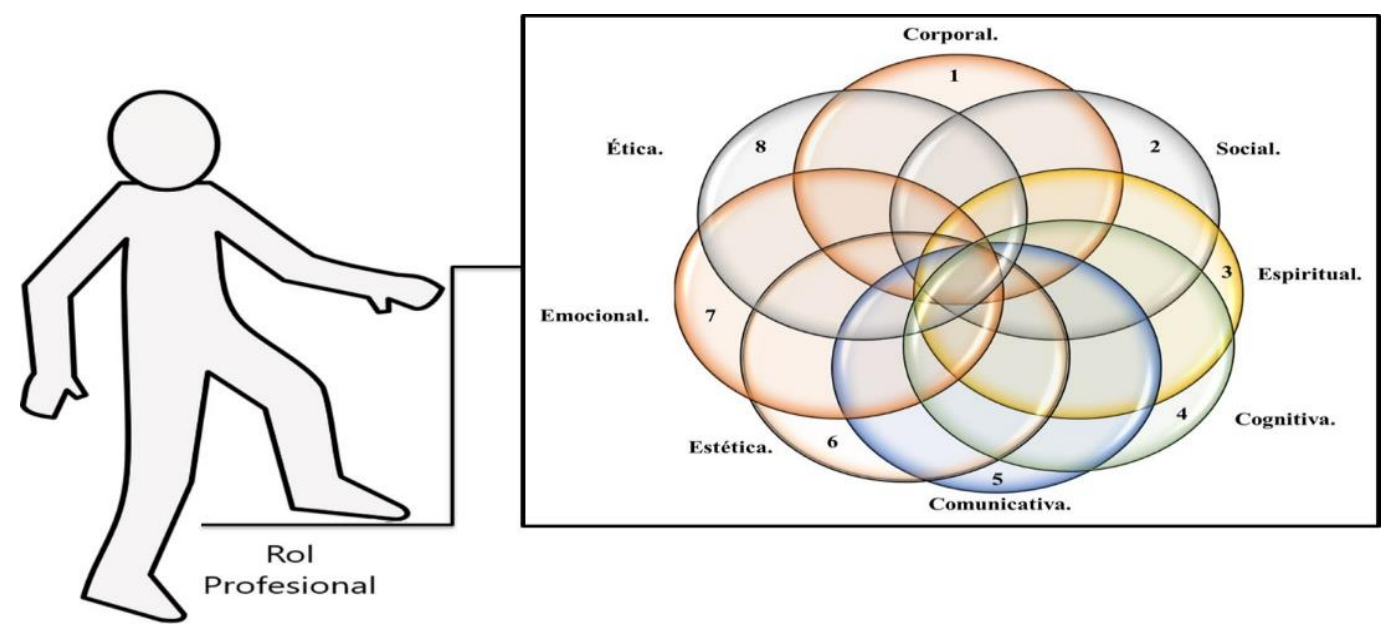

Ilustración 2. Adaptado de: Morales, B. Palencia, J. (2020). Reflexiones del cuidado enfermero en tiempos de covid-19.

La propuesta está encaminada a integrar aspectos orientadores para la aplicación de acciones de cuidado en los niveles de prevención del proceso salud-enfermedad para la dimensión espiritual, buscando mantener un equilibrio biopsicosocial, disminuyendo el espacio de vacuidad de las demás dimensiones entre ellas la espiritual. Ya que, es una de las más importantes para el mantenimiento de la salud, para la capacidad de afrontamiento de la enfermedad, siendo un pilar fundamental para la toma de decisiones (25). 
En la atención en salud los profesionales de enfermería deben plantear estrategias (26) en entornos multidinámicos, multiétnicos, multidisciplinarios, multiculturales, en el abordaje del cuidado espiritual. Razón por la cual, el reto es pasar de lo teórico a lo práctico, desde el respeto mutuo por las creencias que cada individuo elige. La dimensión espiritual es un tema que no se ha profundizado en la práctica, lo cual fue evidenciado en los antecedentes, a pesar de la importancia del mismo; es una dimensión que la profesión de enfermería contempla desde la ciencia y el arte del cuidado. La misión de los profesionales de enfermería es desarrollar procesos de atención a esta dimensión con el conocimiento científico, la humanización e integralidad que caracterizan el cuidado. Existen instrumentos (27-29) para la valoración (30) de la dimensión espiritual que se han aplicado más en atención a 11 personas con cuidados paliativos. Sin embargo, no se han contextualizado en las diferentes áreas del saber donde interviene enfermería. La formación enfatiza el cuidado holístico, contempla diagnósticos de enfermería para el desarrollo del plan de cuidados, en relación a los objetivos e intervenciones, para la promoción de la salud y prevención de aspectos que tienen estrecha relación con las necesidades de las otras dimensiones, sin tener conciencia de la intervención en lo espiritual (Sin que ello signifique religión), afectando directamente en el proceso salud - enfermedad por la integración que hay entre ellas $(31,32)$.

En consecuencia, dentro de los grandes retos que el equipo humano de enfermería tiene, es con el cuidado de la dimensión espiritual, por ser tan íntima en cada persona, por reconocer en sí mismo y en el otro una verdad autónoma de su vivencia espiritual, porque sólo en casos extremos de sufrimiento se expresa la necesidad del alma-espíritu, que trasciende lo corpóreo. Otro reto, es retomar distintas visiones en la formación de los enfermeros como: la teología, el enfoque espiritual en lo antropológico, sociológico para profundizar algunos aspectos de relaciones y funciones del hombre en la sociedad. En síntesis, es vital la interacción e intersección del que cuida, con quién recibe el cuidado, en el entorno desde el proceso saludenfermedad (33).

Es por ello, que la reflexión de este artículo nace de una revisión de antecedentes bibliográficos, evidenciando interés y reflexión de los profesionales de enfermería frente a la temática; detallado en las siguientes tablas:

\begin{tabular}{llcc}
\hline Años & Booleano / Frase & $\begin{array}{c}\text { Tipos de } \\
\text { Fuente }\end{array}$ & $\begin{array}{c}\text { Resultados de } \\
\text { búsqueda }\end{array}$ \\
\hline 2019 a 2014 & $\begin{array}{l}\text { Dimensión espiritual } \\
\text { AND cuidado paliativo } \\
\text { AND Enfermería }\end{array}$ & Artículos & 13 \\
\hline 2013 a 2002 & Total & $\mathbf{1 9}$ \\
\hline 2019 a 2014 & $\begin{array}{l}\text { Cuidado paliativo AND } \\
\text { Bioética } \\
\text { Dimensión espiritual }\end{array}$ & Artículos & 12 \\
\hline 2013 a 2002 & & & 8 \\
\hline
\end{tabular}

\begin{tabular}{|c|c|c|c|c|}
\hline Años & Booleano / Frase & $\begin{array}{c}\text { Tipos de } \\
\text { Fuente }\end{array}$ & $\begin{array}{c}\text { Resultados } \\
\text { de } \\
\text { búsqueda }\end{array}$ & Observaciones \\
\hline 2019 a 2014 & \multirow{2}{*}{$\begin{array}{c}\text { (Dimensión } \\
\text { espiritual AND } \\
\text { cuidado paliativo } \\
\text { AND } \\
\text { Enfermería) y } \\
\text { (Cuidado } \\
\text { paliativo AND } \\
\text { Bioética AND } \\
\text { Dimensión } \\
\text { espiritual) }\end{array}$} & \multirow{2}{*}{$\begin{array}{c}\text { Libros } \\
\text { Tesis doctorales } \\
\text { Monografías }\end{array}$} & 11 & Publicados por: \\
\hline \multirow[t]{2}{*}{2013 a 2002} & & & 9 & $\begin{array}{l}\text { 1. Sociología } \\
\text { 2. Filosofía } \\
\text { 3. Enfermería } \\
\text { 4. Antropología } \\
\text { 5. Psicología }\end{array}$ \\
\hline & & Total & 20 & \\
\hline
\end{tabular}




\section{ANTECEDENTES BIBLIOGRÁFICOS DE LA DIMENSIÓN ESPIRITUAL}

\section{NECESIDADES}

"Significado, Esperanza, Alivio, Paz interior" $(34,35)$.

“Fe. Dios. Creencia. Amor. Tranquilidad. Armonía. Meditación. Bondad. Espíritu. Transcendencia” $(14,36)$.

"La religión. Conocimiento interno de la persona" (37).

"Reconciliación con la propia vida. Decir adiós. Creer en un ser supremo. Compañía" (18,38,39).

"Hambre misma de orden, de libertad, de obediencia, de responsabilidad, de igualdad, de jerarquía, de honor, de castigo, de libertad de opinión, de seguridad, de riesgo, de verdad, de propiedad privada y de propiedad colectiva" (40).

“De sentido, de reconciliación, de sentirse perdonado, simbólico ritual, de soledad y de gratuidad” (41-43).

Búsqueda de sentido: ¿quién soy?, ¿qué hago aquí?, ¿qué sentido tiene mi vida? $(44,45)$.

\section{INTERVENCIONES}

“Actitudes espirituales como compasión, presencia y hospitalidad" $(5,13)$.

"Estar presente y brindar apoyo cuando el paciente experimente dudas, miedo, sufrimiento, desesperación u otros estados psicológicos difíciles" (18).

"Ofrecer presencia de manera compasiva. La escucha es un acto espiritual porque está impregnado de competencia interior" (45).

"Logoterapia de Víctor Frankl en la que el hombre expresa sus preocupaciones más profundas a través del diálogo" (21).

"Desarrollar relaciones basadas en un auténtico cuidado confiable" (5).

"La oración o la meditación". Tener un respeto profundo por la dignidad humana, con apertura a las diferentes creencias, o a la ausencia de las mismas, admitir al ser humano en su realidad trascendente" $(11,46)$.

"Ayuda para el control del enfado. Clarificación de valores. Dar esperanza. Escucha activa. Facilitar el duelo. Facilitar la expresión del sentimiento de culpa. Facilitar la meditación. Facilitar el perdón. Facilitar la práctica religiosa. Potenciación de la autoestima. Potenciación de la conciencia de sí mismo. Potenciación de la socialización" $(30,31)$.

"Hablar, escuchar, orar y realizar lecturas religiosas o de naturaleza espiritual; muchas veces esta intervención se puede limitar a "estar" con el paciente en silencio y cuidar que el paciente pueda tener momentos de privacidad espiritual" $(14,15,47)$.

"Respetar y apoyar los valores, creencias, y conexiones importantes, y el más ambicioso, dar de sí mismo" (48).

"Crear una atmósfera de afectividad y empatía donde el paciente sienta confianza y logre exteriorizar sus necesidades espirituales. Dispone el tiempo y la actitud necesarios para reafirmar su propia perspectiva espiritual" (49).

Propuesta

1. La dimensión espiritual es motivo de vida para cada persona, se piensa, se vive, se auto reflexiona. Se hace relevante la parte afectiva, el amor como necesidad humana, el cual fluye en cada persona desde lo individual, la pareja, la familia, los amigos, expresado desde la ternura, la atención así mismo y al otro. El amor conlleva dependencia afectiva, lo que le hace vulnerable y sensible a depender del otro emocionalmente; el profesional de enfermería motiva la comunicación con su entorno, con los seres amados de manera respetuosa, generando espacios de confianza y solidaridad. La persona para poder estar en armonía interior, tiene metas, proyectos que le llevan a buscar estabilidad, al trascender en equilibrio interior se promueve la integralidad espiritual.
2. Los profesionales de enfermería se preocupan por reconocer el entorno donde viven las personas, familias y comunidades. Este entorno relacionado con lo expresado por Madeleine Leininger como "conjunto de hechos, situaciones y experiencias en escenarios físicos, ecológicos, sociopolíticos, o culturales" (23). Tienen en cuenta las creencias religiosas, espirituales que conlleven a entender la dimensión espiritual dentro de la integralidad.

Religioso: motivar lectura de acuerdo a credo religioso, leer, tener imágenes, escritos de acuerdo a necesidades religiosas, motivar la interacción con personas según creencias personales. En los hospitales y clínicas tener una capilla o sitio de oración el cual conozcan las personas, motivar a orar, a hacer silencios, reflexionar en diferentes momentos. En las Instituciones 
educativas se deben implementar cátedras donde se haga reflexión desde lo espiritual: jardines infantiles, primaria, bachillerato, universidades y sitios laborales; se deben dejar espacios para el contacto con la naturaleza, cátedras donde se eduque con diferentes visiones desde la dimensión espiritual; enfermería tiene un rol educativo preponderante en las instituciones, promoviendo el cuidado espiritual desde el incremento de valores, prácticas donde se aprenda a escuchar al otro, el juego como expresión de armonía interior.

Espiritual: Estar en el aquí y ahora, motivar contacto con la naturaleza (en lo posible), hacer silencios, contacto con el yo interior (cerrar ojos, respirar de manera abdominal, mente en calma), escucha activa de la naturaleza; abrazar un árbol, motivar el uso de otras alternativas de autocuidado como: técnicas de relajación y meditación, yoga, uso de la musicoterapia, sol terapia. Cuidado a los animales, a la naturaleza; enfermería hace uso de las terapias alternativas y se apropia, motivando a ampliar visiones frente al cuidado de la dimensión espiritual. En la medida que una persona reciba formación, puede tener herramientas que llevan a cambiar su realidad; tener una visión más amplia de la espiritualidad, genera cambios de pensamiento, promueve desde todos los contextos, otras formas de vivir la vida.

Cultural: Integrar estructura social y cultural, los saberes de cada persona, entender la necesidad espiritual, tener en cuenta sugerencias de autocuidado y cuidado, ser sensible al sentir interior del otro, generar espacios de acercamiento a través del dialogo en respeto, armonía. Enfermería muestra interés, por trabajar al interior de las comunidades en el fortalecimiento de la dimensión espiritual, generando una comunicación en el respeto de las creencias, valores, normas, leyes, con el fin de mejorar procesos de salud.

3. El cuidado del cuerpo, los cuidados que hace cada persona por mantenerse en forma hacen parte del eje de salud, sin embargo, es importante analizar las actitudes de las personas en la vida, la búsqueda de su sentido de vida. Desde lo espiritual enfermería puede motivar a la persona a entender su proyección, su sentido de justicia, a pensar de manera más positiva frente al entorno que se vive, a analizar valores, creencias, a cambiar estructuras de pensamientos inflexibles; motivar a buscar el bien común. Desde las instituciones educativas promover espacios de cuidados físicos, pero también del mundo interior, enseñar a gestionar las emociones en los diferentes contextos de vida.

4. Enfermería tiene en cuenta en su quehacer profesional: La escucha activa, ser buen dialogante, flexible en su forma de pensar, creativo, empático, sociable, cálido, tener conocimiento científico, gusto por el trabajo en equipo, trato digno y humanizado; permitir el diálogo de saberes con el equipo de trabajo, la persona, la familia, la comunidad, en los diferentes contextos de su ciclo vital. Sabe entender los valores, las creencias, las prácticas de la cultura individual, entendiendo que el cuidado espiritual se hace desde la autonomía personal. Enfermería hace tratamiento a la persona, pareja, familia y grupos en la dimensión espiritual, acogiendo las necesidades espirituales, teniendo en cuenta los contextos culturales, sociales de cada persona.

\section{CONCLUSIONES}

La dimensión espiritual es uno de los ejes centrales en el estudio del ser humano, es el encuentro entre lo tangible y lo intangible, entre lo que representa el mundo y lo que no es. Cuando se piensa en la espiritualidad, el ser humano se ve representado en un ser superior que te guía interiormente, que te mueve a seguir empoderado en cada instante de la vida. La espiritualidad en el cuidado del profesional de enfermería, representa el ir al encuentro de dos almas, de dos espíritus, de dos seres humanos unidos por una verdad superior que nos muestra la vulnerabilidad, la fragilidad, la fuerza de salir adelante en un contexto de salud que puede llevar a trascender.

La espiritualidad representa la oportunidad para reencontrar ese mundo interior, ese yo inconsciente que se pierde con la obstinación del mundo egoíco, donde prima lo externo, el cuerpo, el conocimiento, lo social, sin detenerse la persona a reflexionar en ese mundo interno que la equilibra, que la conecta con su paz interior, con su SER, con su todo. El profesional de enfermería tiene un mundo que trascender en ese acompañamiento con el otro ser, en el cual son espejos que se representan desde una mirada de reciprocidad, de solidaridad, de respeto, de amor, de armonía; cuando enfermería trasciende su cuidado desde lo espiritual, se está moviendo en la esencia del SER, en la esencia de lo sublime.

La espiritualidad desde el cuidado de enfermería es sinfonía, música, es el amor por ese otro que siente, piensa, sufre, cuestiona, adolece, en donde los múltiples diagnósticos de enfermedad priman sobre la esencia de la vida. En este mundo moderno, los valores, las normas, las leyes, llevan a repensar el cuidado de la dimensión espiritual, donde la escucha, la mirada, el contacto hacen parte de ese reencuentro con el mundo interior, donde los seres humanos paciente- enfermera se unen en simbiosis para transformar las culturas, los mitos, los tabús, las barreras, que han llevado a que se pierda esa esencia espiritual del yo interno, del yo silencioso, del yo meditativo, del yo ético que puede generar esperanza en un estado de vulnerabilidad, que nos lleva a enfermar.

La espiritualidad representa ese encuentro respetuoso, responsable, amable, amoroso del profesional de enfermería con la persona vulnerable, con el ser humano que pasa por un momento de crisis, con la comunidad que pierde su sentido de vida, con una sociedad que lo único que reclama es encontrar su esencia, su SER a través de diferentes visiones, que cada vez la alejan de sí misma, sin entender que lo interno, es la respuesta a ese empoderamiento externo; cuando el espíritu no evoluciona, no trasciende, no mira más allá de lo evidente, ese mundo externo enceguece más y más, llevando a que prioricen el cuidado de lo corporal.

\section{CONFLICTO DE INTERES \\ Ninguno}

\section{FINANCIACIÓN \\ Ninguno}

AGRADECIMIENTOS

Dr. Guillermo Rafael Cantú Quintanilla. 


\section{REFERENCIAS}

1. Olea Gutiérrez CV, Berumen Burciaga LV, Zavala Perez IC. Modelo de cuidado de enfermería para la mujer con cáncer de mama a través de la integración de la dimensión espiritual. R. d. Enfermería, Editor. 2012;6(3) Disponible en: http://eneenfermeria.org/ojs/index.php/ENE/article/view/205

2. García Moyano LM, Pellicer García B, Arrazola Aerdi O. La ética del cuidado, sustento de la bioética enfermera. Revista Latinoamericana de Bioética. 2015;16(30-1):72-79. Disponible en: http://dx.doi.org/10.18359/rlbi.1442

3. Germán Zurriaráin R. Los límites éticos: ¿avances o retroceso? Persona y Bioética. 2017; 21(2): 233-242. Disponible en: DOI:10.5294/pebi.2017.21.2.4. http://www.scielo.org.co/scielo.php?script=sci_abstract\&pid=S0123-31222017000200233

4. Vicente Plasencia Llanos. Ser humano: un proyecto inconcluso Reflexiones filosófico-teológicas sobre la Antropología. Editorial Universitaria Abya-Yala. 2017, p 150

5. Ortega Jiménez M, Ojeda Vargas MG, Ortiz Domínguez A, Guerrero Castañeda RF. Spirituality and nursing care. Humanísticas, Editor. 2016. ISSN: 2395-7972. Disponible en: https://www.ricsh.org.mx/index.php/RICSH/article/view/86/452

6. Wood Molina T, Rivas Riveros E. Conflictividad ética en enfermeras/os de unidades de cuidados críticos en un hospital del sur de Chile. E. U. 2017;14(4):224-234, Disponible en: https://doi.org/10.1016/j.reu.2017.09.002

7. Arouca S. La historia natural de las enfermedades. Revista Cubana de Salud Pública.2018;44(4). Disponible en: https://www.redalyc.org/jatsRepo/214/21458870018/html/index.html

8. Organización Mundial de la Salud (OMS). Alivio del dolor y tratamiento paliativo en cáncer. Informe de un Comité de expertos. Organización Mundial de la Salud, Serie de Informes Técnicos 804: Ginebra, Suiza: 1990, p. 11-2. Disponible en: https://apps.who.int/iris/handle/10665/41759

9. Jim H, Purnell J, Richardson S, Golden Kreutz D, Andersen B. Measuring meaning in life following cancer. S. Link, Editor. 2006;15:1355-1371. Disponible en: https://link.springer.com/article/10.1007/s11136-006-0028-6

10. Schiappacasse Cocio G, González Soto P. Validation of Meaning in Life Scale modified to evaluate the spiritual dimension in a Chilean and Latin-American cancer population on palliative care. G. M. Oncología, Editor. 2016;15(3):121-127. Disponible en: https://doi.org/10.1016/j.gamo.2016.05.004. http://www.sciencedirect.com/science/article/pii/S166592011630027X

11. Terceño López C. Vivencia de la atención espiritual del equipo de enfermería en un hospital público. Universitat rovira i virgili. Tarragona. España. 2017, p 276

12. Sánchez Herrera B. Dimensión espiritual del cuidado en situaciones de cronicidad y muerte. Surgen luces de esperanza para acoger mejor el reto de la totalidad, tras años de investigación en enfermería. Aquichan. 2004; 4(1):2027-5374. Disponible en: https://aquichan.unisabana.edu.co/index.php/aquichan/article/view/44.

13. Jasso-Soto ME, Pozos-Magaña MG, Olvera-Arreola SS, et al. Análisis del bienestar espiritual de los pacientes cardiópatas hospitalizados en una institución de salud. Rev Mex Enf Cardiol. 2014;22(3):98-105. Disponible en: https://www.medigraphic.com/cgi$\mathrm{bin} / \mathrm{new} /$ resumen.cgi?IDARTICULO=59602

14. Pinedo Velázquez MT, Jiménez Jiménez JC. Care nursing the spiritual dimension of the patient. Systematic review. C. d. digital. 2017, 21(48): 110-118. Disponible en: http://dx.doi.org/10.14198/cuid.2017.48.13

15. Sandoval P, Rangel N, Allende S, Ascencio L. Concepto de espiritualidad del equipo multidisciplinario de una unidad de cuidados paliativos: un estudio descriptivo. Rev. Psicooncología. 2014;11(2-3):333-344. Disponible en: https://revistas.ucm.es/index.php/PSIC/article/download/47392/44425/

16. Uribe Velásquez SP, Lagoueyte Gómez MI. "Estar ahí", significado del cuidado espiritual: la mirada de los profesionales de enfermería. A. E. Enfermería. 2014; 32(2):261-270. Disponible en: http://www.scielo.org.co/scielo.php?pid=S012145002014000200010\&script=sci_abstract\&tIng=es

17. Gutiérrez Carmona A, Alday MC, Calderón CC. Validación de la versión en español de la escala Perspectiva Espiritual de P. Reed. Citado en Rev. Cuba Enf. 2020;36(1):1-12. Disponible en: https://www.medigraphic.com/cgibin/new/resumen.cgi?IDARTICULO=97035

18. Suárez EE. Ética en el Cuidado de Enfermería. R. V. Enfermería, 2015;2(2). Disponible en: http://190.169.94.12/ojs/index.php/rev_venf/article/download/10216/9997

19. Alarcón Soca I. Necesidades espirituales de los pacientes terminales del servicio de medicina en el Hospital Nacional Dos de Mayo. T. U. Marcos. 2009. Disponible en: https://www.semanticscholar.org/paper/Necesidades-espirituales-de-los-pacientes-del-de-enSoca/be5db8c902c9ff168ed95448fe971c02601b179c

20. Ortega Galán ÁM, González de Haro MD. El valor de la dimensión espiritual en el final de la vida desde la perspectiva de los profesionales de enfermería. M. Paliativa. 2013;23(2):93-98. Disponible en: https://doi.org/10.1016/j.medipa.2013.09.004 https://www.sciencedirect.com/science/article/abs/pii/S1134248X14000317?via\%3Dihub

21. Hinshaw, D. Spiritual Issues in Surgical Palliative Care. Elsevier. Saunders. 2005; 85(2):257-72. Disponible en: https://pubmed.ncbi.nlm.nih.gov/15833470/

22. Gómez MB. La espiritualidad en el hombre. Desde la perspectiva logoterapéutica de Víctor Frankl. Psicología y Psicopedagogía. Instituto de Investigaciones Psicológicas, Facultad de Psicología y Psicopedagogía. Universidad de El Salvador. 2002. Disponible en: http://p3.usal.edu.ar/index.php/psico/article/view/1244/1580

23. Palomar Palomar C, Ortega A, Ibáñez O. Últimos días. El proceso de morir. En: Pérez E, Medina FJ, editores. Aspectos psicosociales en los cuidados paliativos. Madrid: FUDEN; 2010.

24. Marriner, Tomey. Modelos y teorías en enfermería. Septima Edición. Elsevier Mosby. 2011, 816

25. Morales B. Palencia J. Reflexiones del cuidado enfermero en tiempos de covid-19. Enferm Inv. 2020;5(3):71-78. Disponible en: https://revistas.uta.edu.ec/erevista/index.php/enfi/article/view/914. 
26. Vallés Fructuoso O, Ruiz de Pablo B., Fernández Plaza M., Fuentes Milá V, Martínez Estalella G. Perspectiva de los profesionales de enfermería de unidades de cuidados intensivos sobre la limitación del tratamiento de soporte vital. E. Intensiva., Editor, \& S. E. Coronarias. 2015; 27(4):138-145. Disponible en: https://www.elsevier.es/es-revista-enfermeria-intensiva-142-articulo-perspectivaprofesionales-enfermeria-unidades-cuidados-S1130239916300372

27. Rodríguez Ramírez Y, Acosta Sariego JR, Barrios Osuna I, Morera Pérez M, López Milhet AB. Estado y perspectivas del nuevo modelo ético de enfermería en servicios quirúrgicos de mínimo acceso. H. Médicas. 2016; 16(2):258-272. Disponible en: http://scielo.sld.cu/scielo.php?script=sci_arttext\&pid=S1727-81202016000200006

28. Díaz Heredia Luz Patricia, Muñoz Sánchez Alba Idaly, Vargas Divane de. Confiabilidad y validez del cuestionario de espiritualidad de Parsian y Dunning en versión española. Rev. Latino-Am. Enfermagem [Internet]. 2012; 20(3):559-566. Available from: http://www.scielo.br/scielo.php?script=sci_arttext\&pid=S0104-11692012000300018\&lng=en. https://doi.org/10.1590/S010411692012000300018.

29. Morales-Castillo F.A., Hernández-Cruz M.C., Morales Rodríguez M.C., Landeros Olvera E.A.. Validación y estandarización del instrumento: Evaluación de los comportamientos de cuidado otorgado en enfermeras mexicanas. Enferm. univ. 2016; 13(1):3-11. Disponible en: http://www.scielo.org.mx/scielo.php?script=sci_arttext\&pid=S166570632016000100003\&lng=es. https://doi.org/10.1016/.reu.2015.11.005.

30. Ortiz-Gonçalves Belén, Santiago-Sáez Andrés, Albarrán Juan Elena, Labajo González Elena, Perea-Pérez Bernardo. Elaboración de un cuestionario sobre conocimientos y actitudes de la población madrileña frente al final de la vida. Gac Sanit.2018;32(4):373-376.

Disponible en: http://scielo.isciii.es/scielo.php?script=sci_arttext\&pid=S0213-91112018000400373\&lng=es. Epub 07-Dic2020. http://dx.doi.org/10.1016/j.gaceta.2017.09.009.

31. Reig Ferrer A, Arenas MD, Ferrer Cascales R, Fernández Pascual MD, Albaladejo Blázquez N, Gil M, de la Fuente V. Evaluación del bienestar espiritual en pacientes en hemodiálisis. R. N. Nefrología. 2012;32(6):731-742. doi:10.3265/Nefrologia.pre2012.Apr.11384. https://www.revistanefrologia.com/es-evaluacion-del-bienestar-espiritual-pacientes-articulo-X0211699512002330

32. Pérez García E. Enfermería Y Necesidades Espirituales En El Paciente Con Enfermedad En Etapa Terminal. E. C. Humanizados. 2016;5(2):41-45 Disponible en: http://www.scielo.edu.uy/scielo.php?pid=S2393-66062016000200006\&script=sci_arttext

33. Aarón Muñoz D, Morales Moreno I, Bermejo Higuera JC, Galán González J. La Enfermería y los cuidados del sufrimiento espiritual. Index Enferm. 2014; 23(3): 153-156. doi: http://dx.doi.org/10.4321/S1132-12962014000200008

34. Palencia Sierra JJ, Cantú Quintanilla GR, Reyes López A. Impacto del cuidado paliativo en pacientes pediátricos con cáncer sobre la economía familiar, con seguro popular en el Hospital Infantil de México Federico Gómez, México DF. Universidad Panamericana. 2017. Disponible en: http://biblio.upmx.mx/tesis/157751.pdf

35. Pinedo MT. La humanización de los cuidados: Reto de la enfermería del siglo XXI. Haciendo visible, lo invisible. El papel de enfermería frente al sufrimiento espiritual del paciente. Valencia. Consejo de Enfermería de la Comunidad Valenciana. 2011. Disponible en: http://www.bibliotecadigitalcecova.com/revistas/descargar/revista/libro-155

36. Bai M, Lazenby M. A systematic review of associations between spiritual well-being and quality of life at the scale and factor levels in studies among patients with cancer. J. Medicine. 2015;18(3). Disponible en: https://doi.org/10.1089/jpm.2014.0189.

37. He Gijsberts MJ, Liefbroer Al, Otten R, Olsman E. Spiritual Care in Palliative Care: A Systematic Review of the Recent European Literature. M. Sciences. 2019;7(2):25. Disponible en: https://doi.org/10.3390/medsci7020025.

38. Evangelista Carla Braz, Lopes Maria Emilia Limeira, Costa Solange Fatima Geraldo da, Batista Patricia Serpa de Souza, Batista Jaqueline Brito Vidal, Oliveira Amanda Maritsa de Magalhães. Cuidados paliativos e espiritualidade: revisao integrativa da literatura. Rev. Bras. Enferm. 2016;69(3):591-601. Disponível em: http://www.scielo.br/scielo.php?script=sci_arttext\&pid=S0034$71672016000300591 \&$ Ing=pt. https://doi.org/10.1590/0034-7167.2016690324i.

39. Manchola Camilo, Brazão Elisa, Pulschen Anelise, Santos Marcos. Cuidados paliativos, espiritualidade e bioética narrativa em unidade de saúde especializada. Rev. Bioét. 2016; 24(1):165-175. Disponível em: http://www.scielo.br/scielo.php?script=sci_arttext\&pid=S1983-80422016000100165\&lng=pt. https://doi.org/10.1590/198380422016241118.

40. Sieverson C, Evans V, Florenzano R, Fernández L. ¿Qué Elementos Se Incorporan En Las Evaluaciones De La Espiritualidad Y Religiosidad? Una Revisión De Instrumentos Validados. Editores \& P. Universitaria. 2016;12(2):216-223. Disponible en: http://revistagpu.cl/2016/GPU_junio_2016_PDF/INV_Que_elementos.pdf

41. Weil S. Echar raíces. Editorial Trotta. Segunda edición. 2014: 240. ISBN: 978-84-9879-532-5

42. Torralba Roselló F. La espiritualidad. Editorial Milenio. 2014. ISBN: 13:9788497436021.

43. Alvarado Valle E, Rodríguez Jiménez S, Cárdenas Jiménez M, Pacheco Arce AL. Spiritual care a way to transcend the reason of being of Nursing. Rev Enferm Inst Mex Seguro Soc. 2018;26(1):56-61. Disponible en: https://www.medigraphic.com/pdfs/enfermeriaimss/eim-2018/eim181j.pdf

44. González Rivera JA, Rosario Rodríguez A, Pagán Torres OM. Confirmatory Factorial Analysis of the Personal Spirituality Scale in Puerto Ricans Adults. Interacciones.2018;4(3):153-162 Disponible en: https://pesquisa.bvsalud.org/portal/resource/pt/biblio981368? src $=$ similardocs

45. Lasair, S. A Narrative Approach to Spirituality and Spiritual Care in Health Care. J Relig Health. 2020;59: 1524-1540. https://doi.org/10.1007/s10943-019-00912-9

46. Rosas-Jiménez CA, Díaz-Díaz A. La escucha: elemento fundamental en la atención espiritual del cuidado paliativo. pers.bioét. 2017; 21(2): 280-291. DOI: 10.5294/pebi.2017.21.2.

47. van der Weegen, K., Hoondert, M., Timmermann, M. et al. Ritualization as Alternative Approach to the Spiritual Dimension of Palliative Care: A Concept Analysis. J Relig Health. 2019;58: 2036-2046. https://doi.org/10.1007/s10943-019-00792-z

48. Cuartas Hoyos P, Charry Hernández RL, Ospina Muñoz P, Carreño Corredor S. Spiritual care: A look from the perspective of the model of symptom management and palliative care. R. C. Enfermería. 2019;18:1-17. Disponible en: https://doi.org/10.18270/rce.v18i1.2341 
49. Galvis López MA, Pérez Giraldo B. Revisión de la literatura sobre el concepto "Espiritualidad" aplicado a la práctica de enfermería. R. I. Enfermería. 2013; 3(3):54-56. Disponible en: https://www.enfermeria21.com/revistas/aladefe/articulo/82/revision-de-la-literaturasobre-el-concepto-espiritualidad-aplicado-a-la-practica-de-enfermeria/

50. Morales Ramón F, Ojeda Vargas M. El cuidado espiritual como una oportunidad de cuidado y trascendencia en la atención de enfermería. Salud en Tabasco. 2014; 20(3):94-97. Disponible en: https://www.researchgate.net/publication/334508331_El_cuidado_espiritual_como_una_oportunidad_de_cuidado_y_trascendencia_ en_la_atencion_deenfermeria 